\title{
Stability Analysis of Microgrid Islanding Transients based on Interconnected Dissipative Subsystems
}

\section{Citation for published version (APA):}

Roos, M. H., Nguyen, P. H., Morren, J., \& Slootweg, J. G. (2021). Stability Analysis of Microgrid Islanding Transients based on Interconnected Dissipative Subsystems. IEEE Transactions on Smart Grid, 12(6), 46554667. https://doi.org/10.1109/TSG.2021.3100396

DOI:

10.1109/TSG.2021.3100396

Document status and date:

Published: 01/11/2021

\section{Document Version:}

Accepted manuscript including changes made at the peer-review stage

\section{Please check the document version of this publication:}

- A submitted manuscript is the version of the article upon submission and before peer-review. There can be important differences between the submitted version and the official published version of record. People interested in the research are advised to contact the author for the final version of the publication, or visit the $\mathrm{DOI}$ to the publisher's website.

- The final author version and the galley proof are versions of the publication after peer review.

- The final published version features the final layout of the paper including the volume, issue and page numbers.

Link to publication

\section{General rights}

Copyright and moral rights for the publications made accessible in the public portal are retained by the authors and/or other copyright owners and it is a condition of accessing publications that users recognise and abide by the legal requirements associated with these rights.

- Users may download and print one copy of any publication from the public portal for the purpose of private study or research.

- You may not further distribute the material or use it for any profit-making activity or commercial gain

- You may freely distribute the URL identifying the publication in the public portal.

If the publication is distributed under the terms of Article 25fa of the Dutch Copyright Act, indicated by the "Taverne" license above, please follow below link for the End User Agreement:

www.tue.nl/taverne

Take down policy

If you believe that this document breaches copyright please contact us at:

openaccess@tue.nl

providing details and we will investigate your claim. 


\title{
Stability Analysis of Microgrid Islanding Transients based on Interconnected Dissipative Subsystems
}

\author{
M.H. Roos, Student Member, IEEE, P.H. Nguyen, Member, IEEE, J. Morren, Member, IEEE, \\ and J.G. Slootweg, Senior Member, IEEE
}

\begin{abstract}
To ensure successful islanding of microgrids after a fault has occurred, the transient stability should be analyzed under a set of expected operating conditions during the design and operation of microgrids. Transient stability analysis is conventionally performed with time-domain analysis which is computationally expensive and does not quantify the stability margin. Energy-based methodologies can determine the stability margin, however existing methodologies require significant simplifications to be applied to the microgrid model. The energybased stability analysis methodology proposed in this paper enables the analysis of high-dimension nonlinear microgrid systems and quantification of the stability margin within reasonable time. The performance of the methodology is validated by analyzing a case study microgrid and comparing the results to time-domain analysis and to a state-of-the-art methodology proposed in the literature. The results indicate that the proposed methodology has a significantly lower computational burden and similar accuracy compared to existing energy-based methodologies. The methodology is able to improve the probability of stable islanding of the case study microgrid from $74 \%$ up to $94 \%$ when only optimizing the design, and up to $100 \%$ when optimizing design and control actions.
\end{abstract}

Index Terms-Power system stability, Microgrids, Power system transients, Lyapunov methods, Islanding.

\section{NOMENCLATURE}

\section{Notation}

$\begin{array}{ll}f, g f i, g s i & \begin{array}{l}\text { Fault, grid-feeding islanding, grid-supporting } \\ \text { islanding stages. } \\ \text { Time derivative of } x .\end{array} \\ \dot{x} & \begin{array}{l}\text { Block-diagonal matrix consisting of } A_{i} \forall i= \\ i=1 \ldots I\end{array} \\ & 1 \ldots I . \\ {[a \times b]} & \text { Matrix with } a \text { rows and } b \text { columns. } \\ 0_{a \times b} & \text { Matrix of } a \text { by } b \text { zeros. } \\ d, d c & \text { Design, design and control optimized. }\end{array}$

Model Parameters

$V_{D C} \quad$ Primary DC source voltage.

$R_{d c}, L_{d c}, C_{d c}$ DC-link resistance, inductance, capacitance.

$K_{p p l l}, K_{i p l l}$ Proportional, integral PLL controller gains.

$K_{p c}, K_{i c} \quad$ Proportional, integral current controller gains.

$K_{p v}, K_{i v} \quad$ Proportional, integral voltage controller gains.

$r_{f}, L_{f} \quad$ Inverter-side LCL filter resistance, inductance.

$r_{c}, L_{c} \quad$ Grid-side LCL filter resistance, inductance.

This work has received funding from the European Union's Horizon 2020 research and innovation program under grant agreement No 773717.

M.H.Roos, P.H. Nguyen, J. Morren and J.G. Slootweg are with the Department of Electrical Engineering, Eindhoven University of Technology, Eindhoven, the Netherlands e-mail: m.h.roos@tue.nl

$\begin{array}{ll}C_{f} & \text { LCL filter capacitance. } \\ V_{t r i} & \text { Amplitude of the PWM driver signal. } \\ I_{\text {lim }}, T_{\text {lim }} & \text { Current, torque limit. } \\ \omega_{c} & \text { Droop control low-pass filter bandwidth. } \\ R_{L}, L_{L}, C_{L} & \text { Load resistance, inductance, capacitance. } \\ F_{r}, J & \text { Friction, inertia coefficients. } \\ K_{p \omega}, K_{i \omega} & \text { Proportional, integral speed controller gains. } \\ K_{p t}, K_{i t} & \text { Proportional, integral torque controller gains. } \\ K_{p f} & \text { Proportional, integral flux controller gain. } \\ R_{s}, R_{r} & \text { Stator, rotor resistance. } \\ L_{s}, L_{r}, L_{m} & \text { Stator, rotor, mutual inductance. } \\ R_{l i n e}, L_{\text {line }} & \text { Line resistance, inductance. } \\ V_{n}, \omega_{n} & \text { Nominal voltage, frequency. }\end{array}$

\section{INTRODUCTION}

W HEN a fault occurs nearby a microgrid, the microgrid may transition from grid-connected to islanded operation to continue the supply of (part of) the load in the network [1]-[3]. During the fault-initiated islanding (FII) transient, part of the distributed energy resources (DERs) and load may dropoff, and instability can occur [3]-[5].

The stability characteristics of microgrids differ from conventional power systems e.g. due to their high penetration of inverter-based DERs, coupling between voltage and frequency, and short line length. The stability phenomena in microgrids can be classified into (i) control system (in)stability and (ii) power supply and balance (in)stability [6]. Control system instability generally occurs due to poorly tuned controllers or desynchronization of DERs, while power supply and balanced instability is caused by a (temporary) lack of generation capacity. Both types of stability can occur during or after FII transients.

The transient stability of FII microgrids has been previously analyzed by [3], [4], [7]. The effects of inverter control and the type of load on FII stability is investigated by [4], the impact of inverter control-mode switching delay and the demand/supply ratio is analyzed by [3], and the effect of different fault types is shown by [7]. To prevent instability during or after FII, rigorous transient stability analysis of the FII transient should be performed during the design of a microgrid.

Since the conditions of the resulting microgrids are highly variable due to intermittent (renewable) generation, load variation and structural variation, stability of the FII transient should be analyzed under different operating conditions, fault locations and fault types during the design of the microgrid. However due to the large number of conditions which can 
occur, it may be infeasible to analyze the stability under all conditions in advance. Therefore, on-line FII stability analysis may be continuously performed to prepare for unplanned FII during microgrid operation based on a smaller set of (shortterm) forecast conditions.

To allow optimization of the microgrid design and control actions, the stability of microgrids should be quantified during the stability analysis. However, most large-signal stability analysis of microgrids in the literature is performed with timedomain analysis [8], which only provides an open-form stability verdict (i.e. for a single initial condition) and does not quantify the stability margin. In contrast, energy-based stability analysis provides a closed-form stability verdict and quantifies the stability margin with the domain of attraction (DOA). Several energy-based methodologies have been proposed in the literature based on Brayton-Moser's mixed potential, blockdiagonalized quadratic Lyapunov function (BDQLF), Sum-ofsquares (SOS) programming and Takagi-Sugeno (TS) multimodeling [9]-[12]. Both Brayton-Moser's and BDQLF provide a relatively conservative estimation of the DOA [9], [11]. An advantage of SOS programming is that it can be more accurate (i.e. less conservative) than TS multimodeling when a polynomial Lyapunov function of order higher than two is used [11]. However, this also increases computation time which makes it less suitable for on-line stability analysis. An advantage of TS multimodeling is that the stability of a nonlinear system is determined by analyzing linear matrices, allowing the use of some methodologies intended for linear systems on nonlinear systems [13].

While the analysis of transient stability of conventional power systems can often be based on stability analysis of individual subsystems, the transient stability of microgrids should be analyzed with system-wide nonlinear stability analysis due to the strong coupling between the subsystems and system states [6]. However most of the literature using energybased stability analysis treats the individual components of microgrids e.g. DERs and loads [14], as the application of the aforementioned approaches to systems with a high number of nonlinear terms is infeasible. Some system-wide microgrid stability studies have been performed with simplifications. In [10], the stability of a generic autonomous microgrid is analyzed by reducing the number of nonlinear terms with a simple load model and small phase angle assumption. The stability of a simplified DC microgrid model with a constant power load is analyzed based on energy functions by [11]. Another study is performed by [15] where the stability verdict of an autonomous microgrid is based only on synchronization of the droop controllers.

To enable energy-based stability analysis for highdimension nonlinear microgrids without simplifications, the microgrid system can be divided into a set of interconnected subsystems. Methodologies for the stability analysis of interconnected subsystems have been proposed in the literature, however most approaches proposed in the literature assume that the interconnection between the subsystems is "weak" (i.e. the interactions are relatively small) [13], [16], which makes these approaches not applicable to microgrids due to their strong coupling [6].
A methodology for estimation of the DOA based on nonlinear decoupling is proposed by [17]. The nonlinear decoupling allows the analysis of first-order and second-order quadratic systems from which the DOA can be derived with relatively low computation burden. However, the methodology is suitable for systems with solely quadratic nonlinearities, which is not generally applicable in AC microgrids.

Dissipativity theory enables stability analysis of dynamical systems based on the concept that a system is stable if the total energy of a system decreases over time [18], [19]. A thorough analysis of the generation of energy functions for interconnected linear systems based on dissipativity theory is provided by [20], [21]. However, this has so far not been extended to interconnected nonlinear microgrid systems in the literature.

To enable optimization of the design and control actions of high-dimension nonlinear microgrids based on quantification of the stability margin, this paper proposes an energy-based stability analysis for nonlinear interconnected microgrid systems based on TS multimodeling and dissipativity theory. To apply this analysis to FII transients, the drop-off of DERs and loads is determined with the drop-off analysis methodology also proposed in this paper. The stability analysis methodology is used to optimize the microgrid design and control actions based on the probability of stable islanding, stability margin and cost of a case study microgrid.

The main contributions of this paper are:

1) Proposition of a methodology for estimation of the DOA of high-dimension nonlinear microgrid systems which is used to optimize microgrid design and control actions for stable fault-initiated islanding.

2) Proposition of a methodology to determine the DER and load drop-off during FII transients.

3) Validation of the accuracy and speed of the stability analysis and drop-off methodologies by comparing to timedomain analysis and an existing energy-based methodology.

4) Demonstration of the microgrid design and control action optimization methodology in a FII microgrid case study.

The background theory required for the stability analysis methodology is discussed in the next section. In section III the stability analysis and optimization methodology, dropoff analysis methodology, and case study are described. The validation and case study results are given in section IV and discussed in section V. Finally, conclusions are given in section VI.

\section{THEORY}

\section{A. Autonomous microgrid state-space model}

Microgrids can be modeled in the $d q$ reference frame in nonlinear form $\dot{x}(t)=A(x(t)) x(t)+B_{c}$ as proposed by [10], [22] and shown in Appendix A, where $x(t)$ are the system states, $A(x(t))$ is the nonlinear system matrix and $B_{c}$ is the input. The system can be transformed to an autonomous system $\dot{\tilde{x}}(t)=A(x(t)) \tilde{x}(t)$ with preservation of dynamics by shifting the equilibrium point to the origin where $\tilde{x}(t)=x(t)-x_{e q}$ where $x_{e q}$ is the equilibrium point [23]. 
From this point onward the states after the change of variables will be denoted by $x(t)$ (with exception of Appendix A).

\section{B. Takagi-Sugeno multimodeling}

Nonlinear autonomous systems can be described as TS multimodels of the form $\dot{x}(t)=\sum_{m=1}^{M} h_{m} A_{m} x(t)$, where $M$ is the number of local models and $h_{m}$ are the membership functions [13]. As is evident from the model, a nonlinear system can be represented by a sum of local linear models with the associated membership functions. The local linear models are created by setting the nonlinear terms in the system matrix $A(x(t))$ to constant minimum and maximum values $f_{\text {min }}, f_{\max }$, which can be obtained for different combinations of state values $x$. This modeling approach can be used for nonlinear models containing one-to-one function nonlinearities in the studies domain and has been used for e.g. multiplication, division, exponential and square root nonlinearities [9], [13]. A stability analysis methodology for autonomous nonlinear systems based on this principle was proposed by [9], which is used by many researchers due to its effectiveness and simplicity [10], [24]. However since all possible combinations of $f_{\min }$ and $f_{\max }$ have to be analyzed, the number of linear matrix inequalities (LMIs) which has to be solved is equal to $2^{N L T}$, where $N L T$ is the number of unique nonlinear terms in the system matrix. Therefore, the analysis of models with a high number of nonlinear terms is infeasible.

\section{Domain of attraction}

The DOA of a system is a region around a stable equilibrium point in which the states of the system converge to the equilibrium point over time as defined by (1). Where $\phi\left(t, x_{0}\right)$ is the solution of the autonomous system, with $x(t) \in \mathrm{D} \subset \mathbb{R}^{n}$.

$$
D O A=\left\{x_{0} \in \mathrm{D} \mid \lim _{t \rightarrow \infty} \phi\left(t, x_{0}\right)=0\right\}
$$

Expression (1) is difficult to evaluate analytically, however it is recognized that a system converges to a stable equilibrium point when the total energy of the system decreases over time [23]. A conservative estimate of the size of the DOA described by energy-function $V(x(t))$ can then be made according to (2), where $r$ is a positive scalar, $V(x(t)) \geq 0, V(0)=0$ and $D O A_{\text {est }} \subset D O A$.

$$
\left.D O A_{e s t}=\left\{x(t) \in \mathbb{R}^{n} \mid V(x(t)) \leq r \wedge \dot{V}(x(t))<0\right)\right\}
$$

\section{Interconnected dissipative systems}

The requirements of (2) are fulfilled when a system is dissipative according to the dissipation inequality described by (3) [18], [19]. The energy stored in the system is described by energy-function $V(x(t)) \geq 0$, while the energy supplied to the systems is described by $s(v(t), w(t))$ in (4).

$$
\begin{gathered}
V\left(x\left(t_{k}\right)\right) \leq V\left(x\left(t_{k-1}\right)\right)+\int_{t_{k-1}}^{t_{k}} s(v(t), w(t)) d t \\
s(v(t), w(t))=\left[\begin{array}{c}
v(t) \\
w(t)
\end{array}\right]^{T}\left[\begin{array}{cc}
Q & S \\
S^{T} & R
\end{array}\right]\left[\begin{array}{c}
v(t) \\
w(t)
\end{array}\right]
\end{gathered}
$$

Here, $v(t)$ and $w(t)$ are the input and output of the system, $Q$ and $R$ are symmetric matrices, and $S$ is a real matrix. From this point onward, the time dependencies $(t)$ are no longer noted in equations.

A microgrid consists of multiple subsystems, where the total energy in the system can be described by the sum of the energy-functions of the subsystems and the interconnections can be described as supply functions [21], [25]. As described by [20], [21], a system consisting of interconnected linear subsystems is stable if:

1) The subsystems are dissipative with respect to their supply functions.

2) The interconnections between the subsystems are neutral, which is true for subsystems $i$ and $j$ when $s_{i j}\left(v_{i}, w_{i}\right)+$ $s_{j i}\left(v_{j}, w_{j}\right)=0$.

To test the dissipativity of each subsystem $i \in I$, (5) is solved for each subsystem, where $J_{i}$ are the interconnections of subsystem $i, P_{i}=P_{i}^{T}>0$, and the decision variables are: $P_{i}, Q_{i j}, S_{i j}, R_{i j}$. To ensure that the interconnections are neutral $Q_{i j}=-R_{j i}, S_{i j}=-S_{j i}^{T}$. If subsystem $i$ has a state vector $x$ with length $n$, and input and output state vectors $v$ and $w$ have lengths $o$ and $p$, the sizes of the matrices are: $A_{i, m}^{[n \times n]}, B_{i}^{[n \times o]}, C_{i}^{[p \times n]}, Q_{i}^{[o \times o]}, S_{i}^{[o \times p]}, R_{i}^{[p \times p]}$.

$\left[\begin{array}{cc}I & 0 \\ A_{i, m} & B_{i} \\ 0 & I \\ C_{i} & 0\end{array}\right]^{T}\left[\begin{array}{cccc}0 & P_{i} & 0 & 0 \\ P_{i} & 0 & 0 & 0 \\ 0 & 0 & -Q_{i} & -S_{i} \\ 0 & 0 & -S_{i}^{T} & -R_{i}\end{array}\right]\left[\begin{array}{cc}I & 0 \\ A_{i, m} & B_{i} \\ 0 & I \\ C_{i} & 0\end{array}\right] \prec 0$

Where: $B_{i}=\left[B_{i 1}, \ldots, B_{i J_{i}}\right], C_{i}=\left[C_{i 1}^{T}, \ldots, C_{i J_{i}}^{T}\right]^{T}$

$Q_{i}=\underset{j=1 \ldots J_{i}}{\operatorname{blkdiag}}\left(Q_{i j}\right), S_{i}=\underset{j=1 \ldots J_{i}}{\operatorname{blkdiag}}\left(S_{i j}\right), R_{i}=\underset{j=1 \ldots J_{i}}{\operatorname{blkdiag}}\left(R_{i j}\right)$

The subsystems with neutral interconnections are dissipative in the region where (5) is feasible. As the total energy stored in the system is the sum of the energy in the subsystems, the energy-function is described by (6) [21].

$$
V(x)=\sum_{i=1}^{I} x_{i}^{T} P_{i} x_{i}
$$

\section{E. Stability through fault-initiated islanding transients}

As discussed in [5], the FII transient consists of a sequence of three stages: fault, grid-feeding islanding (GFI) and gridsupporting islanding (GSI). The microgrid is in the fault stage after the fault occurred, after islanding the microgrid is in the GFI stage and the microgrid is in the GSI stage after the DER switched to grid-supporting control mode.

This paper proposes Theorem II.1 to determine whether the system states will converge from the initial conditions to the equilibrium point of the GSI stage. The Theorem is based on the convergence of the states to the equilibrium points during each stage as shown in Fig. 1.

Theorem II.1. Let $D O A_{\text {est }}^{f}, D O A_{\text {est }}^{g f i}, D O A_{\text {est }}^{g s i}$ be the estimated DOAs of the fault, grid-feeding islanding and gridsupporting islanding stages, let $x_{e q}^{f}$ and $x_{e q}^{g f i}$ be the equilibrium points of the fault and grid-feeding islanding stages, and 


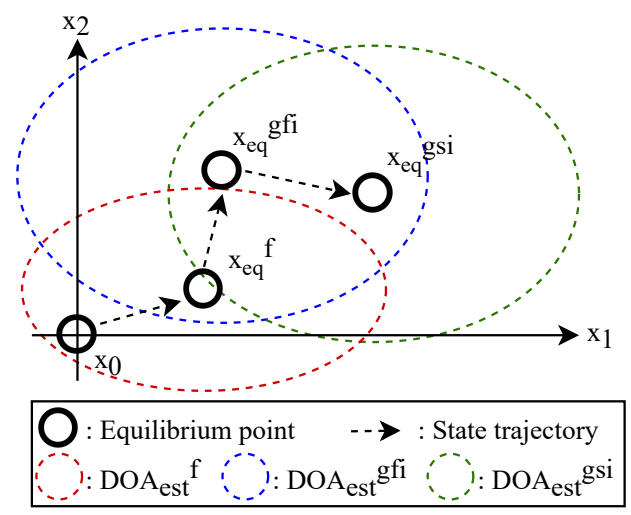

Fig. 1: 2D phase plane representation of example system state trajections from initial conditions $\left(x_{0}\right)$ to equilibrium of the GSI stage $\left(x_{e q}^{g s i}\right)$ according to Theorem II.1.

let $x_{0}$ be the states of the microgrid at the fault instant. If the convergence of the microgrid states is fast so that they are sufficiently close to the equilibrium point at the end of each stage, the microgrid is stable through the FII transient when the following stability verdict expression is true:

$$
\begin{aligned}
\left\{x_{o}\right\} & \in D O A_{\text {est }}^{f} \\
\left\{x_{e q}^{f}\right\} & \in D O A_{\text {est }}^{g f i} \\
\left\{x_{e q}^{g f i}\right\} & \in D O A_{\text {est }}^{g s i}
\end{aligned}
$$

Proof. In case of stable FII, the system states will move from the initial conditions $x_{0}$ towards $x_{e q}^{f}$ during the fault stage if $x_{0} \in D O A_{\text {est }}^{f}$. If the states reach $x_{e q}^{f}$ within the fault stage, the system states will move from $x_{e q}^{f}$ towards $x_{e q}^{g f i}$ during the GFI stage if $x_{e q}^{f} \in D O A_{e s t}^{g f i}$. If the states reach $x_{e q}^{g f i}$ within the GFI stage, the systems states will finally move from $x_{e q}^{g f i}$ towards $x_{e q}^{g s i}$ during the GSI stage if $x_{e q}^{g f i} \in D O A_{e s t}^{g s i}$. This is based on the assumption that the system states converge close to the equilibrium points of each stage, which is valid in microgrids with fast dynamics as shown in Fig. 11.

Note that equilibrium points should exist for each stage of the FII transient to apply Theorem II.1. A lack of an equilibrium point may occur during the fault stage if load devices are modeled as ideal constant power loads [26], [27]. This type of model absorbs an unrealistically high current during the fault stage, while practical load devices would drop-off. However, the load models used in this paper have a constant impedance characteristic or include drop-off behavior, as discussed in Appendix A.

\section{Methodology}

The methodologies proposed in this paper allow stability analysis and optimization of FII microgrids under different conditions based on the theory described in section II. The methodologies are demonstrated with a case study described in this section.

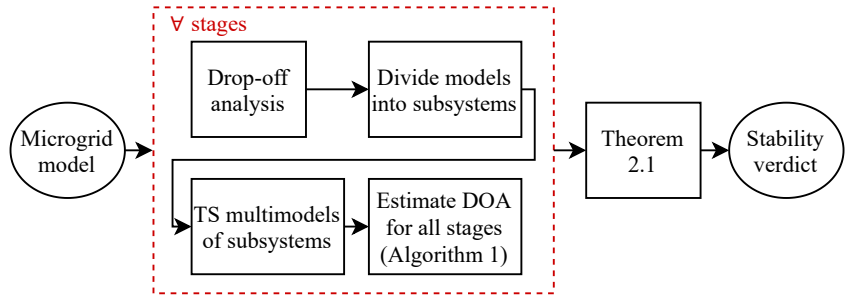

Fig. 2: Stability analysis methodology.

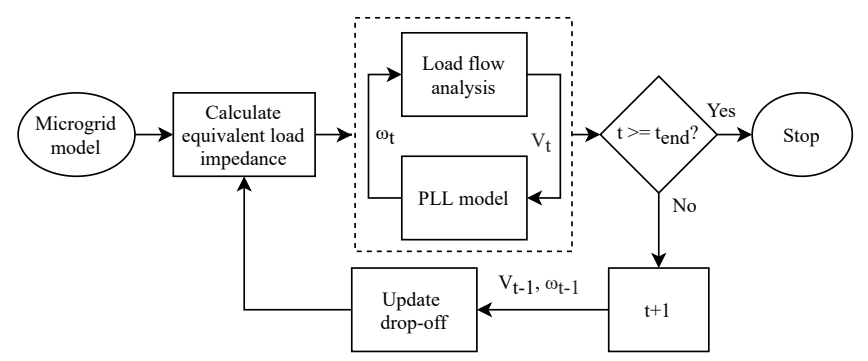

Fig. 3: Drop-off analysis methodology.

\section{A. Stability analysis methodology}

The proposed stability analysis methodology is shown in Fig. 2. The methodology initializes by performing drop-off analysis to determine the operational DERs and loads during each stage of the FII transient as described in section III-A1. Then, the microgrid models of the different FII stages are divided into subsystems to reduce the computational burden of the DOA estimation as described in section III-A2. To allow the theory described in section II-D to be applied, all subsystem models are transformed into TS multimodels as described in section III-A3. The size of the DOA is then estimated for each stage by increasing and decreasing the value of the $f_{\max }$ and $f_{\min }$ terms in the TS multimodels to search the region in which: (i) the subsystems are dissipative and (ii) the interconnections are neutral. As described in section III-A4, the boundary of the estimated DOA is found when (5) is no longer feasible. To analyze the stability through the FII transient, Theorem II.1 is applied as described in section III-A5.

1) Drop-off analysis: The drop-off analysis methodology is used to determine what DERs and loads remain operational during and after the islanding transient. To determine the voltage and frequency in the microgrid over time, a methodology based on load flow calculations and a quasi steady-state PLL model is proposed, so computationally intensive EMT simulations of the full microgrid model are not required.

The methodology shown in Fig. 3 is initialized with all DER and load devices in operation. According to the drop-off behavior at each time-step of the simulation, the equivalent impedance of each load device is calculated. The equivalent impedance of constant current and constant power load devices is described by $Z_{e q, c i}=Z_{0} \frac{V}{V_{n}}$ and $Z_{e q, c p}=Z_{0} \frac{V^{2}}{V n}$ respectively, where $Z_{0}$ is the load impedance at the nominal voltage $V_{n}$.

The voltage in the nodes of the microgrid is determined by performing a load flow analysis similar to the methodology proposed by [28]. However, this methodology cannot 
be directly applied to islanded microgrids during the gridfeeding stage as there is no slack bus or voltage controllers in the network. Therefore, the voltage at the load nodes is firstly calculated according to the branch currents and the equivalent impedance. Secondly, the voltages of other nodes are calculated from the branch currents and the branch-current to bus-voltage as described by [28].

The frequency in the microgrid is determined according to the self-synchronization PLL model proposed for a single DER and load by [29]. However, as there are usually multiple DERs in the network, equivalent DER parameters are determined as described by [30] and used in the PLL model.

The voltage and frequency in the microgrid are determined for every time-step, which allows to check and update the drop-off all DER and load devices at each time step. DER and load devices drop-off when the voltage and/or frequency limits are violated for a duration larger than a time limit set in the controllers of the devices. When a device drops-off, the equivalent load impedance and the PLL model are updated.

2) Divide model into subsystems: As the number of LMIs to be solved with the proposed approach is equal to $\sum_{i=1}^{I} 2^{N L T_{i}}$, where $I$ is the number of subsystems with $N L T_{i}$ the number of unique nonlinear terms in subsystem $i \in I$, the computation time is minimized when the number of unique nonlinear terms are equally divided over the subsystem models. To reach this objective, the DER with the highest power rating and linear loads are considered as the main subsystem. All other (nonlinear) DERs and loads in the network are considered as separate subsystems connected to the main subsystem.

Each interconnected subsystem of the autonomous nonlinear system is described by (7). Where $A_{i}\left(x_{i}\right)$ is the nonlinear system matrix of subsystem $i, B_{i}$ and $C_{i}$ are the input and output matrices of subsystem $i$ to the connected subsystems, $x_{i}$ are the states of subsystem $i$, and $w_{i}$ and $v_{i}$ are the inputs and outputs of subsystem $i$.

$$
\left[\begin{array}{c}
\dot{x_{i}} \\
v_{i}
\end{array}\right]=\left[\begin{array}{cc}
A_{i}\left(x_{i}\right) & B_{i} \\
C_{i} & 0
\end{array}\right]\left[\begin{array}{l}
x_{i} \\
w_{i}
\end{array}\right]
$$

3) Takagi-Sugeno multimodels of subsystems: Before the DOA can be estimated, the nonlinear subsystem models are transformed into TS multimodels, where the nonlinearities take minimum $f_{\min }$ or maximum $f_{\max }$ values. As discussed in II-B, this will lead to a total of $2^{N L T_{i}}$ system matrices $A_{i, m}$ where $N L T_{i}$ is the number of unique nonlinear terms in the system matrix $A_{i}(x)$ of subsystem $i$ and $m \in M_{i}=$ $\left\{1, \ldots, 2^{N L T_{i}}\right\}$ denote the TS multimodels of subsystem $i$. Note that $\sum_{i=1}^{I} 2^{N L T_{i}}$ is much smaller than $2^{N L T}$ in highdimension nonlinear microgrids with multiple subsystems.

4) Estimate domain of attraction: As discussed in section II-D, a linear system is stable if the subsystems are dissipative and the interconnections are neutral. The region in which a nonlinear microgrid is stable can therefore be analyzed by utilizing the linear system matrices $A_{i, m}$ of the TS multimodels. As described by Algorithm 1, this region is determined by initially setting the nonlinear terms $f_{\min }$ and $f_{\max }$ to the equilibrium point $\left(x_{\min }=x_{\max }=0\right)$, and iteratively decreasing and increasing the nonlinear terms while checking
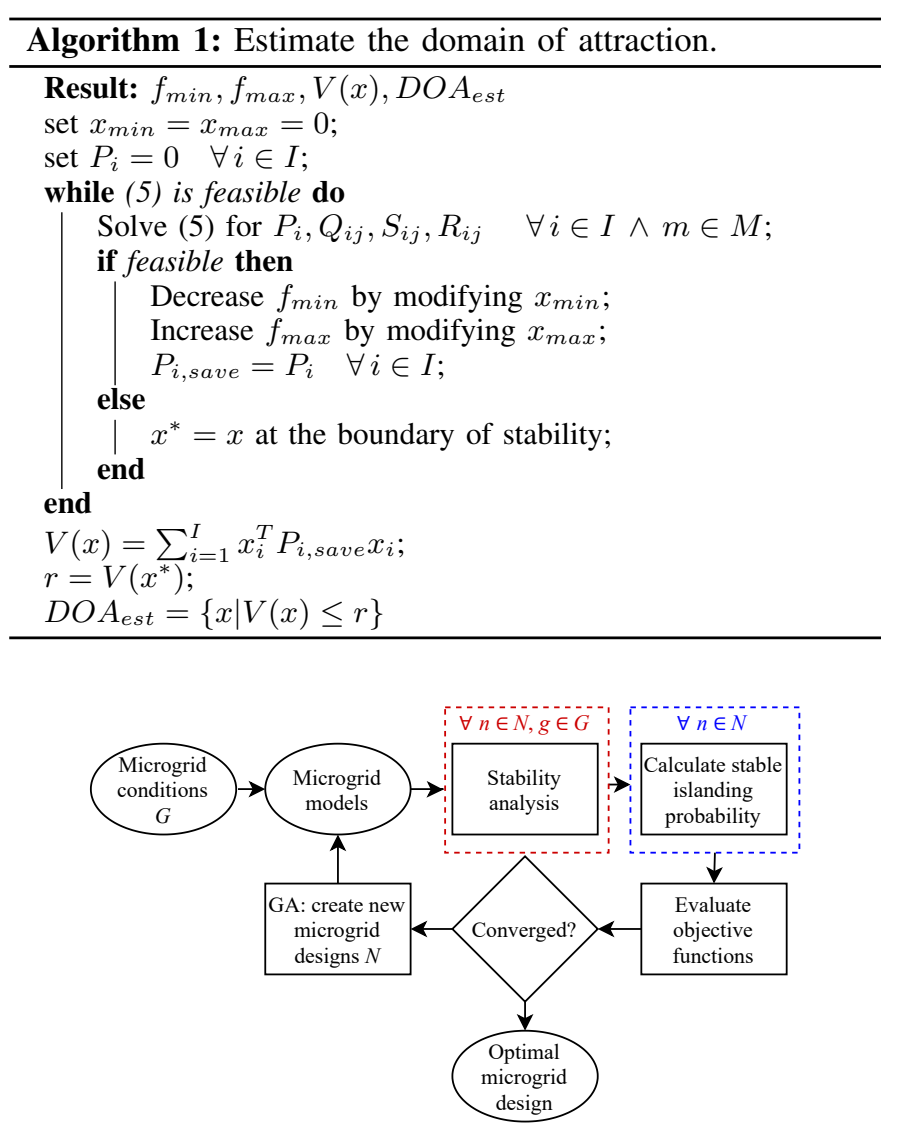

Fig. 4: Microgrid design optimization methodology.

if (5) is feasible $\forall i \in I \wedge m \in M_{i}$. The region found is an estimation of the DOA as the subsystems of the IBMG are dissipative with neutral interconnections.

5) Stability verdict of the fault-initiated islanding transient: When the DOAs of the microgrid during each stage are determined, the stability of the microgrid during the FII transient can be analyzed with Theorem II.1. The distance between the equilibrium points of the different stages, and between the equilibrium points and the initial conditions are determined with (8), where $x_{e q}^{i}$ are the state values at the equilibrium point of stage $i$ and $x_{0}$ are the initial conditions. Then, the conditions of Theorem II.1 can be tested by (9).

$$
\begin{aligned}
\Delta x_{e q}^{i, j}=x_{e q}^{i}-x_{e q}^{j}, \quad \Delta x_{e q}^{i, 0}=x_{e q}^{i}-x_{0} \\
\left\{\Delta x_{e q}^{f, 0}\right\} \in D O A_{e s t}^{f} \\
\left\{\Delta x_{e q}^{g f i, f}\right\} \in D O A_{e s t}^{g f i} \\
\left\{\Delta x_{e q}^{g s i, g f i}\right\} \in D O A_{e s t}^{g s i}
\end{aligned}
$$

\section{B. Microgrid optimization methodology}

The microgrid optimization methodology is initialized by generating microgrid models from an initial population of microgrid designs $N$ with different randomized parameters, reflecting different designs and/or control actions. To generate probabilistic stability results, each microgrid design is analyzed under operating conditions $G$. 


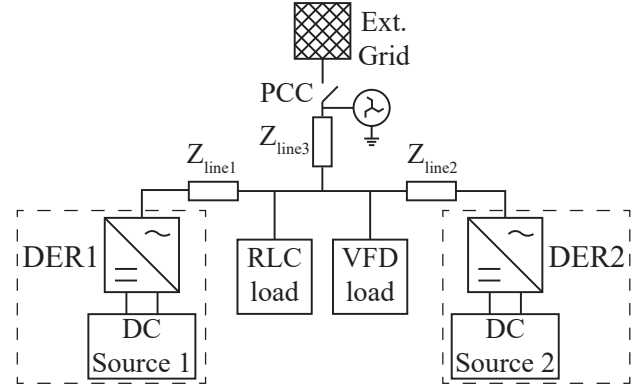

Fig. 5: Case study microgrid.

The resulting microgrid models are analyzed with the stability analysis methodology proposed in section III-A. When the stable operating conditions for each microgrid design are known, the probability of stable islanding for each microgrid design is calculated by using the probability of the operating conditions. At this point the stability of each microgrid $n \in N$ is quantified by the size of $D O A_{\text {est }}$ for each stage and under different conditions, and the probability of stable islanding. These quantities are used to evaluate the objective function of each microgrid design, which in turn is used by a genetic algorithm to generate more optimal populations of microgrid designs. The methodology is executed until the maximum objective function value does not increase for a preset number of iterations (two iterations in this paper).

Multiple objectives are optimized to determine the optimal microgrid design or control actions. Therefore the objective function in (10) is described by the weighted sum of three terms, where $p_{s}$ is the probability of stable islanding, $D O A_{\text {est }, g}^{\text {stage }}$ and $D O A_{\text {req, }}^{\text {stage }}$ are the estimated and required DOA of the stage under conditions $g \in G$, and $C$ is the cost of the microgrid design and control actions required during operation. The required DOA for each stage and condition $D O A_{\text {req, }}^{\text {stage }}$ is defined as the minimum size of the DOA to fulfill the conditions of Theorem 2.1.

$$
f_{\text {obj }}=\alpha_{1} p_{s}+\alpha_{2} \sum_{g=1}^{G} \sum_{\text {stage }=1}^{3}\left(\frac{D O A_{\text {est,g }}^{\text {stage }}}{D O A_{\text {req }, g}^{\text {stage }}}-1\right)-\alpha_{3} C
$$

Genetic algorithms (GAs) are widely used in the literature as they can be applied to many optimization problems with minimal required knowledge about the optimization problem. The GA in this paper generates a population of $N=15$ microgrid designs with different parameters per iteration. The GA initializes the stability analysis and optimization methodology with randomly selected microgrid parameters. To generate a new set of microgrid parameters, the GA selects the five most optimal population members and combines their properties. There is a probability of $5 \%$ that a property of a population member is mutated to a random variable.

\section{Case study}

A case study of the microgrid shown in Fig. 5 is analyzed to demonstrate the effectiveness of the methodologies proposed in this paper. The microgrid consists of a battery energy storage system (DER1), a PV system (DER2), a constant impedance RLC load and a variable frequency drive (VFD) load driving a constant torque load. Both DERs can switch from grid-feeding to grid-supporting control mode. The modeling of the microgrid in the $d q$ reference frame are described in Appendix A. The case study in this paper has two modifications to improve the condition number of the system matrices, making the LMIs accurately solvable. Firstly, the trigonometric terms in the system matrices are replaced by their seventh order Taylor expansion. If the phase angle terms are not replaced, the associated columns of the system $A$ matrices are empty causing the matrix to be ill-conditioned. Secondly, the current and torque limiting sigmoid functions are removed. This reduces the number of nonlinear terms, allowing the estimation of the DOA with the methodology proposed by [9] within reasonable time.

1) Drop-off analysis validation: To validate the drop-off analysis methodology, the voltage, frequency and drop-off after islanding of the case study microgrid are determined with the drop-off analysis methodology and compared to the results of an EMT simulation using the models proposed in [31]. The active power references of both DERs are $P_{1,2}^{*}=5 \mathrm{~kW}$, the VFD load torque is $T_{m}=20 \mathrm{Nm}$, and the parallel RLC load resistance, inductance and capacitance are $R_{L}=9.07 \Omega$, $L_{L}=0.505 \mathrm{H}$ and $C_{L}=201 \mu \mathrm{F}$ respectively.

2) Stability analysis validation: To validate the stability analysis methodology, the DOAs of the microgrid during the fault, GFI and GSI stages are determined with the proposed methodology and compared to the results of the methodologies proposed by [9] and [24], and time-domain analysis results. The active power references of both DERs are $P_{1,2}^{*}=7.5 \mathrm{~kW}$, the VFD load torque is $T_{m}=40 \mathrm{Nm}$, and the parallel RLC load resistance, inductance and capacitance are $R_{L}=15.9 \Omega$, $L_{L}=0.505 \mathrm{H}$ and $C_{L}=20.1 \mu \mathrm{F}$ respectively. The LMIs are constructed and solved using the YALMIP toolbox and MOSEK solver. As discussed before, the computation time of the proposed methodology is dependent on the number of unique nonlinear terms in the system matrix as this determines the number of LMIs which have to be solved. By fixing some of the states that appear in nonlinear terms of the system matrix to zero (the equilibrium point), an analysis of the computation time for different numbers of unique nonlinear terms is performed.

3) Microgrid design and control action optimization: To validate the microgrid design optimization capability of the proposed methodology, the power rating of DER $1\left(S_{1}\right)$ and the DC-link capacitors of both DERs $\left(C_{d c 1}, C_{d c 2}\right)$ are optimized. Subsequently, the active power reference $\left(P^{*}\right)$ and droop control parameters $\left(m p_{1}, n q_{1}\right)$ of DER 1 are optimized to demonstrate the microgrid control action optimization capability. The DER2 solar irradiation probability is described by a Beta distribution with shape parameters $\alpha=4$ and $\beta=2$, while the load probability is described by a lognormal distribution with shape parameters $\mu=0$ and $\sigma=0.69$ [32]. Five samples are taken from both probability distributions and combined to a total of $G=\left\{g_{1}, \ldots, g_{25}\right\}$ microgrid conditions to be analyzed. The DER2 irradiation is such that the power rating can vary between $1 \mathrm{kVA}$ and $10 \mathrm{kVA}$, while the RLC load resistance and VFD load torque vary between $R_{L}=7.94 \Omega$, 


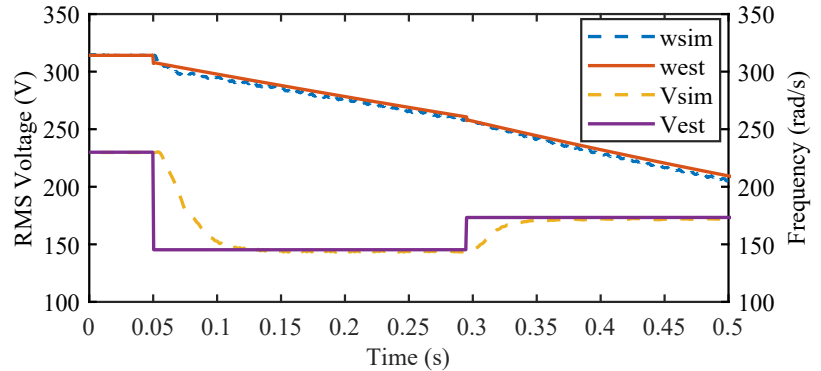

Fig. 6: Frequency and RMS load voltage after switching to islanded operation with DERs in grid-feeding control mode at $t=0.05 \mathrm{~s}$.

$R_{L}=31.7 \Omega$ and $T_{m}=10 \mathrm{Nm}, T_{m}=60 \mathrm{Nm}$ respectively. During the microgrid design optimization, the droop control parameters of DER1 and DER2 are $m p_{1}=\frac{8.125}{S_{1}}, n q_{1}=\frac{0.1 \pi}{S_{1}}$ and $m p_{2}=\frac{9.75}{S_{2}}, n q_{2}=\frac{0.12 \pi}{S_{2}}$ respectively, while the active power references are equal to $75 \%$ of nominal power. The fault and GFI stages both have a fixed duration of 0.2 seconds. The cost of the DER1 power rating and DC-link capacitors is equal to $1 € / \mathrm{VA}$ and $10^{5} € / \mathrm{F}$ and are constrained to a maximum of $100 \mathrm{kVA}$ and $10 \mathrm{mF}$ respectively.

To analyze the impact of the optimization parameters on the solution, the cost and performance of microgrids after design optimization $\left(\operatorname{Var} x^{d}\right)$, and after design and control optimization $\left(\operatorname{Var} x^{d c}\right)$ with different optimization parameters according to table I are compared.

\section{RESUlts}

\section{A. Drop-off analysis validation}

The frequency and load (RMS) voltage in the case study microgrid resulting from EMT simulations and drop-off analysis before and after switching to islanded operation are shown in Fig. 6. After switching to islanded operation at $t=0.05 \mathrm{~s}$, both the voltage and frequency in the network decrease as there is a generation deficiency and the load is dominantly capacitive. Around $t=0.3 \mathrm{~s}$, the VFD drops-off and the voltage increases. The computation time of the EMT simulation is 288 seconds, while the computation time of the drop-off analysis methodology is 0.86 seconds. The voltage, frequency and drop-off in the microgrid over time determined by the drop-off analysis methodology are very similar to the results of the EMT simulation, while the computational burden is significantly smaller. This allows the drop-off analysis methodology to be used in iterative algorithms such as the stability analysis and optimization methodology proposed in this paper.

\section{B. Stability analysis validation}

To visualize $D O A_{\text {est }}^{g s i}$ and time-domain analysis results, twodimensional projections are generated of the results of the proposed methodology (DOA1), [9] (DOA2), [24] (DOA3) and

TABLE I: Objective function parameter variations during sensitivity analysis.

\begin{tabular}{cccccccc}
\hline & Var1 & Var2 & Var3 & Var4 & Var5 & Var6 & Var7 \\
\hline$\alpha_{1}$ & $10^{4}$ & $10^{6}$ & $10^{2}$ & $10^{4}$ & $10^{4}$ & $10^{4}$ & $10^{4}$ \\
$\alpha_{2}$ & $10^{4}$ & $10^{4}$ & $10^{4}$ & $10^{6}$ & $10^{2}$ & $10^{4}$ & $10^{4}$ \\
$\alpha_{3}$ & 1 & 1 & 1 & 1 & 1 & 10 & 0
\end{tabular}

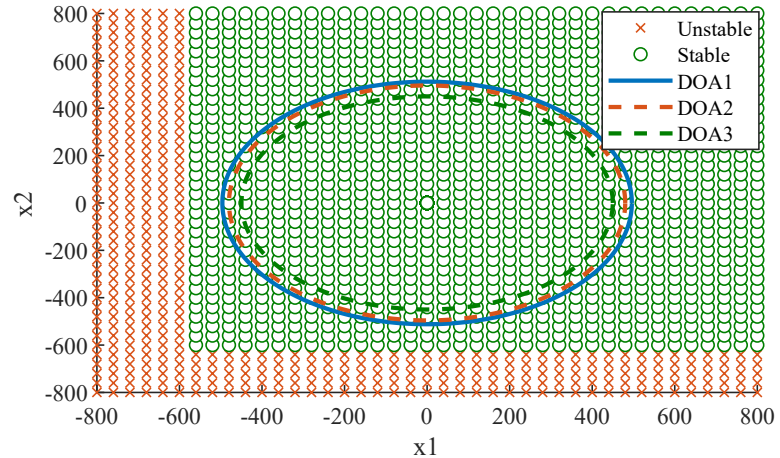

Fig. 7: $D O A_{\text {est }}^{g s i}$ of DC-link voltage states $\left(x_{1}, x_{2}\right)$ with DClink capacitances of $500 \mu \mathrm{F}$ determined with the proposed method (DOA1), [9] (DOA2), [24] (DOA3) and time-domain analysis (x,o).

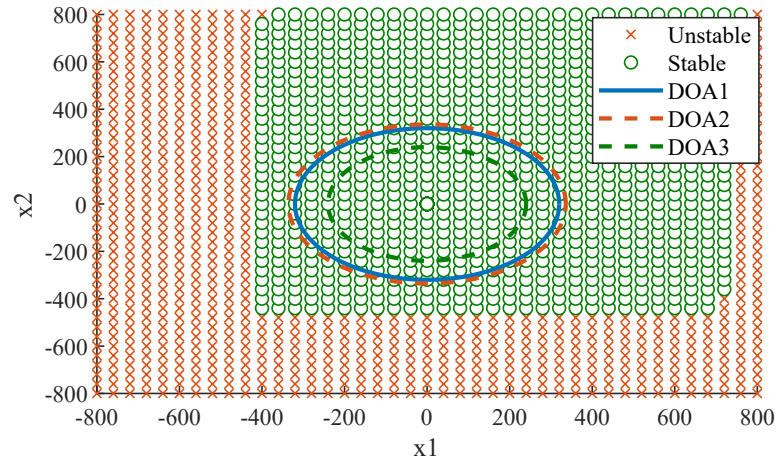

Fig. 8: $D O A_{\text {est }}^{\text {gsi }}$ of DC-link voltage states $\left(x_{1}, x_{2}\right)$ with DC-link capacitances of $20 \mu \mathrm{F}$ determined with the proposed methodology (DOA1), [9] (DOA2), [24] (DOA3) and time-domain analysis (x,o).

the time-domain analysis. The projection of the maximum DClink voltage deviation from the GSI stage equilibrium point of both DERs with a DC-link capacitance of $C_{d c}=500 \mu \mathrm{F}$ and $C_{d c}=20 \mu \mathrm{F}$ are shown in Fig. 7 and 8 respectively. The maximum reactive power deviation of DER1 and phase angle deviation of DER2 from the GSI stage equilibrium point with the original and three times larger droop control parameters are shown in Fig. 9 and 10 respectively. Projections onto the mentioned states are chosen as they occur in nonlinear terms of the system matrix, and deviations of these variables can cause power balance and control system instability [5], [6]. The maximum state deviations from the stable equilibrium points determined by the proposed methodology and timedomain analysis are given in table II.

A comparison of the computation times of the methodology proposed in section III and [9] for different numbers of unique nonlinear terms in the system matrix is shown in table III. Table III also shows the computation time of [24] in case of $N L T=14$, as variation of $N L T$ is not straightforward. The computation time of the proposed methodology is significantly smaller than [9] as the number of LMIs which have to be

TABLE II: Maximum state deviation from the equilibrium point during different stages determined by proposed approach/time-domain analysis.

\begin{tabular}{lccc}
\hline State & Fault & GFI & GSI \\
\hline$V_{d c 1}(\mathrm{~V})$ & $544 / 570$ & $608 / 630$ & $496 / 590$ \\
$V_{d c 2}(\mathrm{~V})$ & $496 / 570$ & $608 / 630$ & $512 / 620$ \\
$\left|\theta_{1}-\theta_{2}\right|(\mathrm{rad})$ & $1.26 / 1.57$ & $2.34 / 2.51$ & $0.164 / 0.173$
\end{tabular}




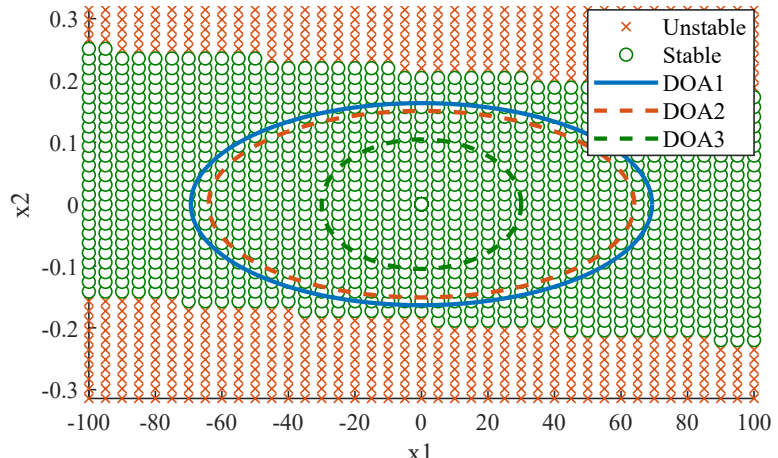

Fig. 9: $D O A_{\text {est }}^{g s i}$ of DER1 reactive power $\left(x_{1}\right)$ and DER2 phase angle $\left(x_{2}\right)$ with original droop control parameters determined with the proposed methodology (DOA1), [9] (DOA2), [24] (DOA3) and time-domain analysis $(\mathrm{x}, \mathrm{o})$.

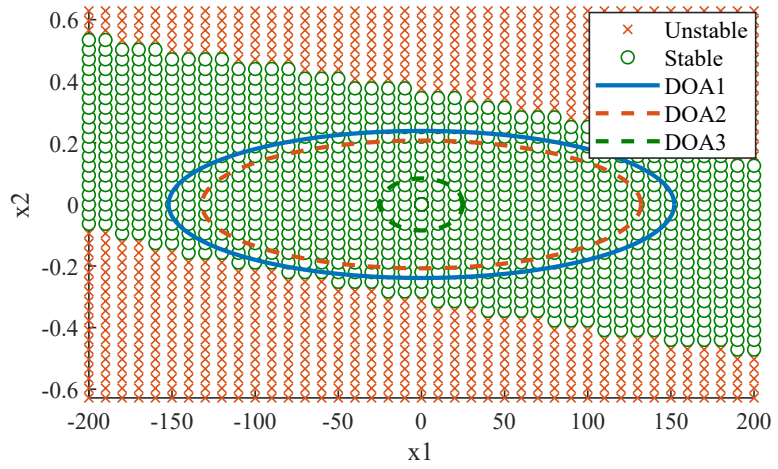

Fig. 10: $D O A_{e s t}^{g s i}$ of DER1 reactive power $\left(x_{1}\right)$ and DER2 phase angle $\left(x_{2}\right)$ with larger droop control parameters determined with the proposed methodology (DOA1), [9] (DOA2), [24] (DOA3) and timedomain analysis $(\mathrm{x}, \mathrm{o})$.

solved and the number of unknowns per LMI is considerably smaller. The computation time of the proposed methodology is also smaller than [24] as the total number of unknowns which have to be solved is smaller.

To validate whether Theorem II.1 can be applied to nonlinear microgrids, the convergence of the states of the microgrid during each stage is analyzed with time-domain analysis as shown in Fig. 11. The states of the microgrid converge close to their equilibria within $0.2 \mathrm{~s}$, which allows Theorem II.1 to be used.

\section{Microgrid design and control action optimization}

The resulting properties and parameters of microgrid optimization with the different parameters in table I are shown in table IV, where (U) is the unoptimized microgrid design which is also used for validation of the stability analysis.

TABLE III: Number of LMIs to be solved and computation time of solving a single iteration with different numbers of unique nonlinear terms being varied. $N L T$ : unique nonlinear terms.

\begin{tabular}{llccccc}
\hline Method & \multirow{2}{*}{ Property } & \multicolumn{5}{c}{$N L T$} \\
\cline { 3 - 7 } & & 3 & 6 & 9 & 12 & 14 \\
\hline \multirow{2}{*}{ Proposed } & LMIs & 6 & 12 & 24 & 48 & 80 \\
& Time (s) & 0.126 & 0.205 & 0.396 & 0.714 & 1.03 \\
{$[9]$} & LMIs & 8 & 64 & 512 & 4096 & 16384 \\
{$[24]$} & Time (s) & 2.18 & 18.87 & 187.1 & 1640 & 7098 \\
& Time (s) & - & - & - & - & 3.84
\end{tabular}

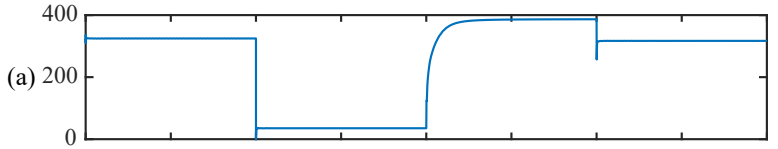

(b)
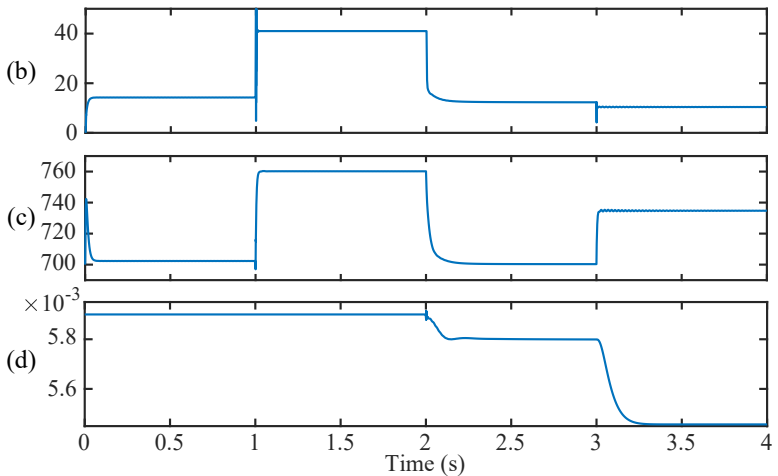

Fig. 11: Convergence of microgrid states during a FII transient with the fault at $t=1 \mathrm{~s}$, islanding at $t=2 \mathrm{~s}$ and DERs control-mode switching at $t=3 \mathrm{~s}$. (a):load voltage (V). (b):DER1 output current (A). (c):DER1 DC-link voltage (V). (d):phase angle (rad).

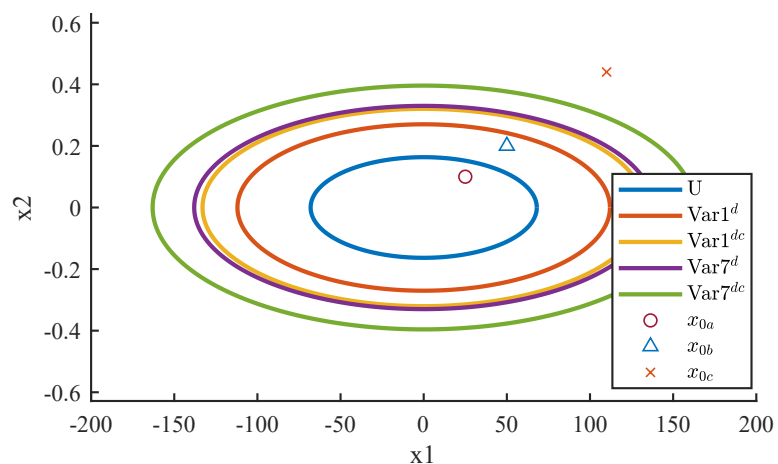

Fig. 12: DO $A_{\text {est }}^{g s i}$ of DER1 reactive power $\left(x_{1}\right)$ and DER2 phase angle $\left(x_{2}\right)$ for unoptimized (U), design optimized $\left(\operatorname{Var} 1^{d}, \operatorname{Var} 7^{d}\right)$, and design and control optimized $\left(\operatorname{Var} 1^{d c}, \operatorname{Var} 7^{d c}\right.$ ) case study microgrid designs.

The average computation time of a single iteration of the optimization methodology shown in Fig. 2 is 926 seconds, while on average 6 iterations were required until the microgrid design and control actions converged, resulting in an average computation time of 5556 seconds.

$D O A_{\text {est }}^{g s i}$ of the unoptimized (U), two design optimized $\left(\operatorname{Var} 1^{d}, \operatorname{Var} 7^{d}\right)$, and two design and control optimized (Var1 ${ }^{d c}$, $\left.\operatorname{Var} 7^{d c}\right)$ microgrids are projected onto the reactive and phase angle systems states are shown in Fig. 12. Time domain simulations are performed with initial conditions $x_{0 a}, x_{0 b}$ and $x_{0 c}$ as shown in Fig. 12, all designs are stable for $x_{0 a}$, only the unoptimized design is unstable for $x_{0 b}$ and all designs are unstable for $x_{0 c}$.

The load voltage, phase angle and DER DC-link voltages of the $\mathrm{U}, \operatorname{Var} 1^{d c}, \operatorname{Var} 7^{d c}$ and $\overline{\operatorname{Var} 7^{d c}}$ microgrid designs during a FII transient are compared in Figs. 13, 14, 15 and 16. Microgrid design $\widetilde{\operatorname{Var} 7^{d c}}$ is equal to design $\operatorname{Var} 7^{d c}$ with droop parameter optimization constraints $m p \geq 0.1$ and $n q \geq 0.1$. The irradiation of DER2 is $5 \mathrm{~kW}$, the VFD load torque is $T_{m}=30 \mathrm{Nm}$, and the RLC load resistance, inductance and capacitance are $R_{L}=15.9 \Omega, L_{L}=0.505 \mathrm{H}$ and $C_{L}=$ $200.6 \mu \mathrm{F}$ respectively. The DC-link voltage of DER1 of design 


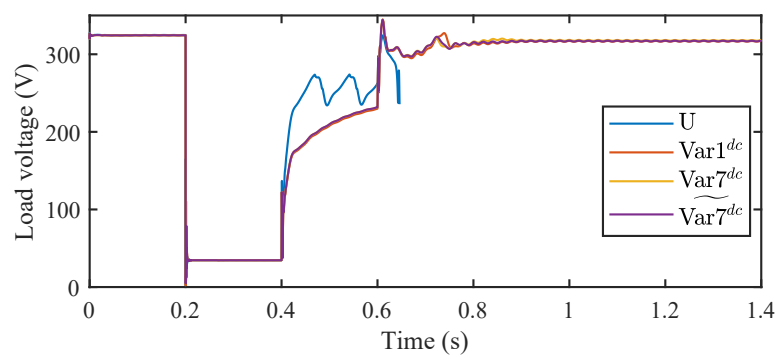

Fig. 13: Load voltage magnitude during a FII transient with the fault at $t=0.2 \mathrm{~s}$, islanding at $t=0.4 \mathrm{~s}$ and DERs control-mode switching at $t=0.6 \mathrm{~s}$.

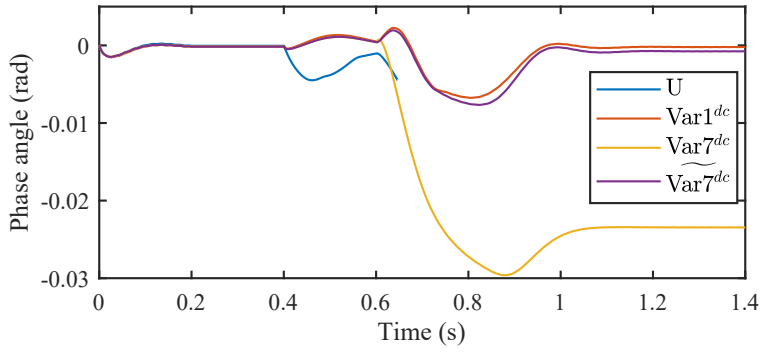

Fig. 14: Phase angle between DER1 and DER2 during a FII transient with the fault at $t=0.2 \mathrm{~s}$, islanding at $t=0.4 \mathrm{~s}$ and DERs controlmode switching at $t=0.6 \mathrm{~s}$.

U goes to zero during the GSI stage, causing DC-link voltage instability and a singularity in the time-domain solution, which is reflected in a distorted load voltage. The optimized designs converge to stable equilibrium points. The DC-link voltage deviation of design $\operatorname{Var} 1^{d c}$ is larger than $\operatorname{Var} 7^{d c}$, which indicates that design $\operatorname{Var} 7^{d c}$ is more stable. However, design $\operatorname{Var} 7^{d c}$ has a larger phase angle difference between the DERs during steady-state, which causes circulating currents. This indicates that the improvement in transient performance of design $\operatorname{Var} 7^{d c}$ has causes deterioration in steady-state performance. This is mitigated in design $\widetilde{\operatorname{Var} 7^{d c}}$, while the DC-link voltage deviations remain small.

\section{DISCUSSION}

The proposed stability analysis methodology enables estimation of the DOA of each FII stage by utilizing TS multimodels to search for a domain in which the microgrid subsystems are dissipative with neutral interconnections. The methodology shows a significant reduction of the computational burden compared to existing TS multimodeling approaches, since the

TABLE IV: Comparison of unoptimized (U) and optimized (Varx) case study microgrid designs with different optimization parameters. $p_{s}^{d}$ : probability of stable islanding of design optimized microgrid. $p_{s}^{d c}$ : probability of stable islanding of design and control optimized microgrid.

\begin{tabular}{lcccccccc}
\hline Design & $p_{s}^{d}$ & $p_{s}^{d c}$ & $\begin{array}{c}\text { Cost } \\
(\mathrm{k} €)\end{array}$ & $\begin{array}{c}C_{d c 1,2} \\
(\mathrm{mF})\end{array}$ & $\begin{array}{c}S_{1} \\
(\mathrm{kVA})\end{array}$ & $\begin{array}{c}m p_{1} \\
(\mathrm{~V} / \mathrm{kW})\end{array}$ & $\begin{array}{c}n q_{1} \\
(\mathrm{rad} / \mathrm{s} / \mathrm{kVAr})\end{array}$ & $\begin{array}{c}P_{1}^{*} \\
(\mathrm{~kW})\end{array}$ \\
\hline $\mathrm{U}$ & 0.743 & 10.1 & 0.500 & 10.0 & 0.813 & 0.0314 & 7.50 \\
$\mathrm{Var1}$ & 0.814 & 0.960 & 6.32 & 1.80 & 5.96 & 0.301 & 0.117 & 4.00 \\
$\mathrm{Var} 2$ & 0.882 & 0.981 & 12.9 & 1.03 & 12.7 & 0.142 & 0.103 & 2.58 \\
$\mathrm{Var} 3$ & 0.755 & 0.815 & 3.90 & 0.869 & 3.73 & 0.160 & 0.160 & 2.92 \\
Var4 & 0.852 & 0.980 & 15.9 & 3.20 & 15.3 & 0.305 & 0.0677 & 4.28 \\
Var5 & 0.770 & 0.860 & 6.46 & 1.00 & 6.26 & 0.249 & 0.146 & 1.02 \\
Var6 & 0.683 & 0.738 & 2.47 & 0.720 & 2.33 & 3.29 & 0.0576 & 0.339 \\
Var7 & 0.936 & 1.00 & 17.0 & 8.30 & 15.3 & 0.305 & 0.0138 & 2.66
\end{tabular}

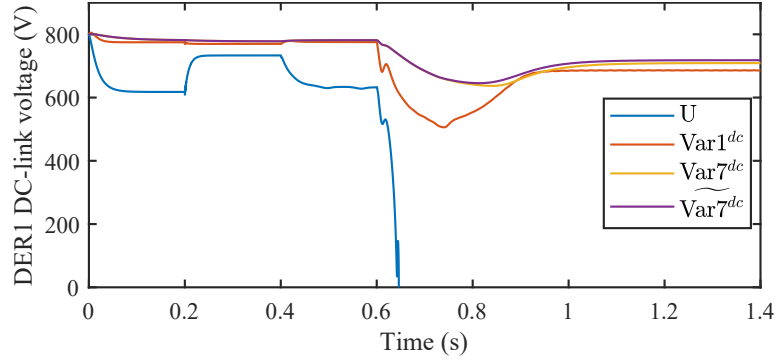

Fig. 15: DER1 DC-link voltage during a FII transient with the fault at $t=0.2 \mathrm{~s}$, islanding at $t=0.4 \mathrm{~s}$ and DERs control-mode switching at $t=0.6 \mathrm{~s}$.

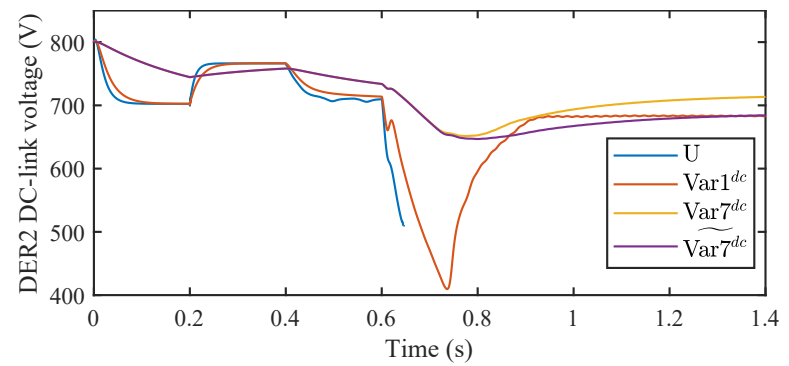

Fig. 16: DER2 DC-link voltage during a FII transient with the fault at $t=0.2 \mathrm{~s}$, islanding at $t=0.4 \mathrm{~s}$ and DERs control-mode switching at $t=0.6 \mathrm{~s}$.

required number of LMIs to be solved is significantly lower and the number of unknowns per LMI is also smaller. The results indicate that the methodology can estimate the DOA of a nonlinear microgrid system with an accuracy similar to stateof-the-art TS multimodeling approaches such as [9], which in turn is similar to SOS programming with a polynomial of order 2 [11]. The reduction in computational burden is smaller compared to approaches based on Lur'e problem [24]. However, the proposed approach more accurately estimates the DOA of the case study microgrid, especially for states associated with the higher-order phase angle nonlinearities.

The reduced computational burden allows iterative optimization during design and operation of microgrids based on the probability of stable islanding and the stability margin. Both design and control optimization have a significant impact on the probability of stable islanding in the case study. The microgrid design after design optimization shows increase probability of stable islanding and size of the DOA. As shown in table IV, increasing the objective function parameter $\alpha_{1}$ mainly increases the probability of stable islanding while also increasing the size of the DOA and cost, increasing $\alpha_{2}$ mainly increases the size of the DOA while also increasing the probability of stable islanding and cost, and increasing $\alpha_{3}$ mainly decreases the cost while also decreasing the probability of stable islanding and size of the DOA. Additionally, the results show that even without cost considerations the probability of stable islanding could not be increased to $100 \%$ in the microgrid design phase. After control and design optimization the $\operatorname{Var} 1^{d c}$ design has a higher probability of stable islanding than both $\operatorname{Var} 1^{d}$ and $\operatorname{Var} 7^{d}$, while the cost is equal to design $\operatorname{Var} 1^{d}$. Design $\operatorname{Var} 7^{d c}$ has a higher cost than $\operatorname{Var} 1^{d c}$, but is stable under all conditions. These results show the effectiveness of the optimization algorithm, and the importance of optimization during both planning and operation of the microgrid. 
The stability during the resynchronization and reconnection of the microgrid has not been considered in this paper. The proposed methodology can be used to analyze the stability during this stage, however the changes of the state values during the resynchronization transient are generally much smaller than during the (fault-initiated) islanding transients. Therefore, small-signal analysis can be applied for stability analysis and controller design as shown by [33].

The case study of this paper considers a microgrid in the $d q$ reference frame. However, $d q$ reference frame models cannot directly take unbalanced loading or structure into account which may be present in practical microgrids. To allow the analysis of unbalanced systems, the microgrid may either be described by the sequence components [34] or a dynamics phasor model [35]. The application of the methodology proposed in this paper on sequence component and dynamic phasor models will be treated in future research.

Time-domain analysis e.g. Fig. 11 indicated that the states of the case study microgrid are close to the equilibrium point at the end of each FII stage, which allows Theorem II.1 to be used to generate a stability verdict. Future research will determine when it is possible to use Theorem II.1 based on the convergence rate and/or time constants of the system to omit the requirement for time-domain analysis.

\section{CONCLUSION}

This paper proposes an energy-based stability analysis methodology for high-dimension nonlinear microgrid models based on the analysis of dissipativity and neutral interconnections of microgrid subsystems modeled as TS multimodels. The results show that the methodology allows accurate estimation of the DOA while having a relatively low computation time. To enable application the proposed stability analysis methodology to FII transients, a drop-off analysis methodology is proposed which is accurate and has a significantly lower computation time than EMT simulations. A case study of a microgrid is performed in which the design and control actions of the DERs are optimized based on the probability of stable islanding, stability margin and cost.

Future research should treat the application of the proposed methodology on unbalanced microgrids and the development of conditions under which the relaxed stability verdict of Theorem II.1 can be applied.

\section{APPENDIX A}

\section{CASE STUDY MODELS}

\section{A. DER and load device models}

The grid-feeding and grid-supporting DER models considered in this paper are described by (11) and (12) respectively, where $v_{o d}, v_{o q}$ and $i_{o d}, i_{o q}$ are the $d q$ components of the node voltage and current respectively [5]. The RLC and VFD load models are described by (13) and (14), while the line model is described by (15). Temporary VFD drop-off occurs when $V_{d c}<420 \mathrm{~V}$ with a hysteresis of $30 \mathrm{~V}$, while permanent VFD drop-off occurs when $\omega_{m}=0$ [31].
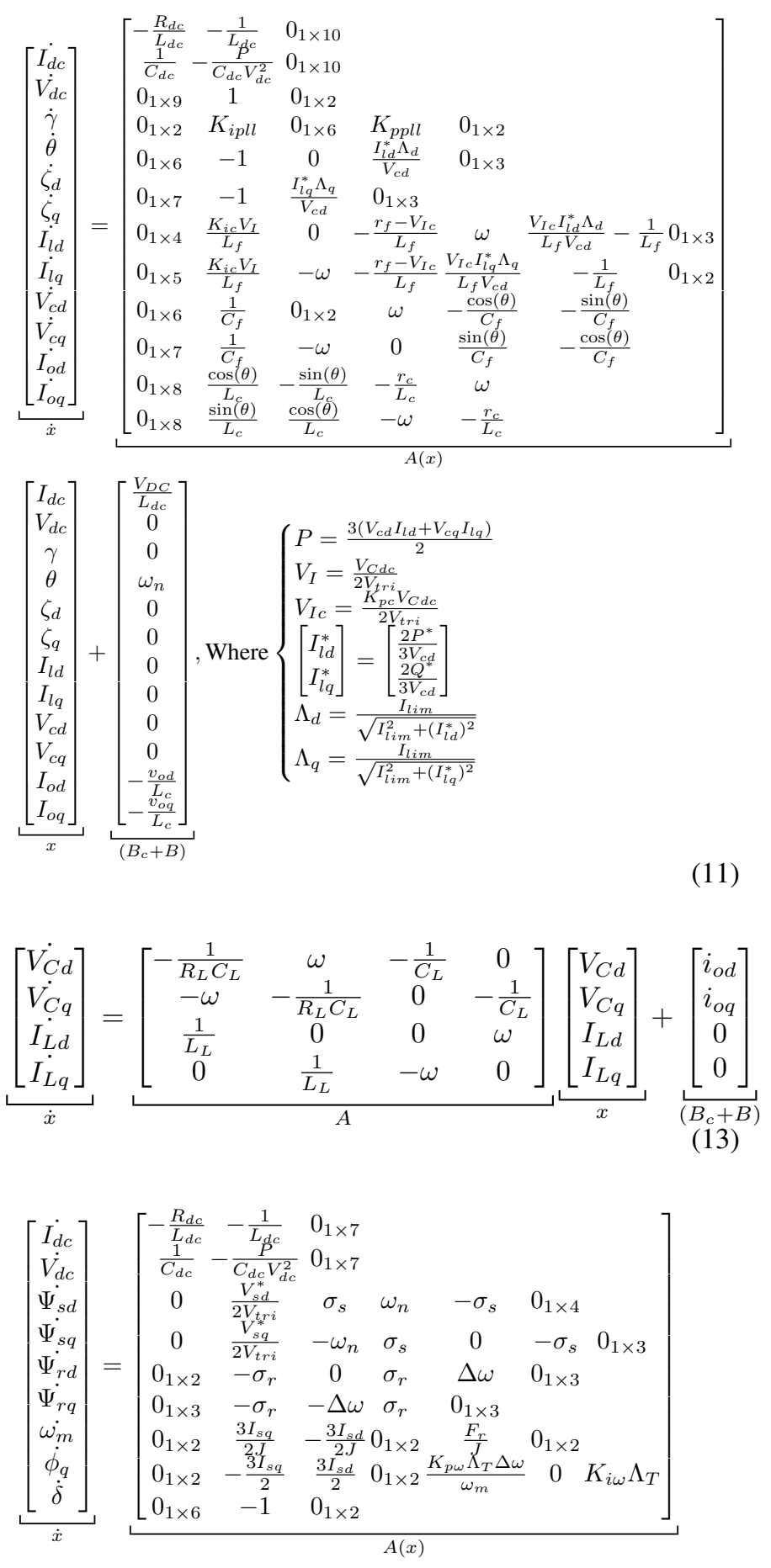

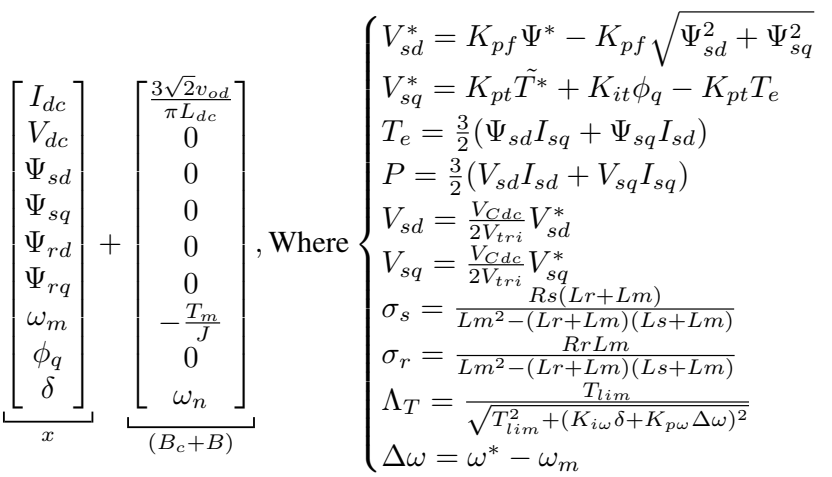




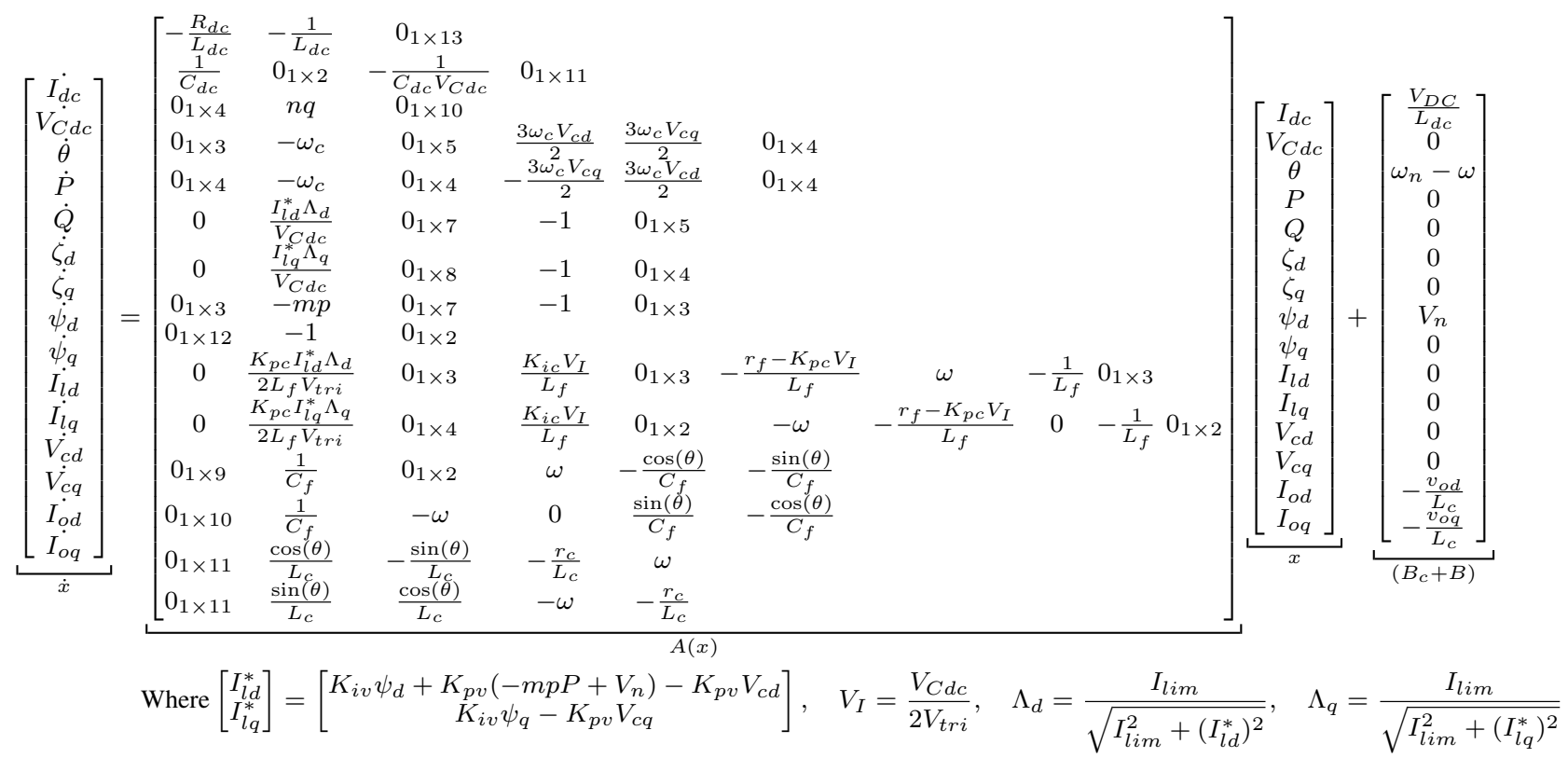

$$
\underbrace{\left[\begin{array}{c}
I_{\text {lined }} \\
I_{\text {lineq }}
\end{array}\right]}_{\dot{x}}=\underbrace{\left[\begin{array}{cc}
-\frac{R_{\text {line }}}{L_{\text {line }}} & \omega \\
-\omega & -\frac{R_{\text {line }}}{L_{\text {line }}}
\end{array}\right]}_{A} \underbrace{\left[\begin{array}{c}
I_{\text {lined }} \\
I_{\text {lineq }}
\end{array}\right]}_{x}+\underbrace{\left[\begin{array}{c}
\frac{v_{\text {oid }}-v_{\text {ojd }}}{L_{\text {line }}} \\
\frac{v_{\text {oiq }}-v_{\text {ojq }}}{L_{\text {line }}}
\end{array}\right]}_{\left(B_{c}+B\right)}
$$

Where $v_{o i d}, v_{o i q}$ and $v_{o j d}, v_{o j q}$ are the $d q$ components of the voltage at node $i$ and $j$ respectively.

\section{B. Microgrid models}

The devices models in the last subsection are expressed as $\dot{x}=A(x) x+\left(B_{c}+B\right)$, where $A(x)$ is the (nonlinear) system matrix, $x$ are the states, $B_{c}$ is a constant input and $B$ are interconnection terms in (7) i.e. the node voltage and/or current. The complete microgrid model of each stage can therefore be developed in the form $\dot{x}=A(x) x+b_{c}$ by connecting the inputs and outputs of the device models that are operational during each FII stage. The alignment of the $d q$ reference frame is chosen depending on the stage of the microgrid. During the fault, the $d q$ reference frame is aligned with the external grid to which the microgrid is connected, therefore the frequency of the $d q$ reference frame is equal to the external grid frequency ( $\omega=\omega_{n}=100 \pi$ ). During the GFI and GSI stages the $d q$ reference frame is aligned with the phase angle of DER1. The frequency is therefore determined by the PLL of DER1 during the GFI stage $(\omega=\dot{\theta})$ and by the inverse droop controller of DER1 during the GSI stage $\left(\omega=n_{1} q Q_{1}\right)$.

To perform stability analysis on the complete microgrid models, the models are transformed into autonomous microgrid models of the form $\dot{\tilde{x}}=A\left(\tilde{x}+x_{e q}\right) \tilde{x}$ as discussed in section II-A. The transformed models can be used for the methodology described by [9] and can be rewritten in Lur'e form $\dot{\tilde{x}}=A \tilde{x}+D h(\tilde{x})$ for the methodology described by [24].

\section{Divide into subsystems}

The stability analysis methodology proposed in this paper requires the microgrid model to be described as interconnected subsystems. As discussed in section III-A2, the computational burden is minimized when the nonlinearities are evenly divided over the subsystems. Subsystem $i=1$ consists of DER1, the RLC load, and lines 2 and 3. Subsystem $i=2$ consists of DER2 and line1, while subsystem Subsystem $i=3$ consists of the VFD load.

As lines 1 and 2 is in series with the output of DER1 and DER2, these lines can be modeled by adding the line impedance to $r_{c}$ and $L_{c}$. The input voltage of DER1, DER2 and the VFD load is then equal to the RLC load voltage $v_{o d}=V_{C d}, v_{o q}=V_{C q}$. The input current of the RLC load is then equal to the sum of the current injected by DER1, DER2 and line 3 , and the current absorbed by the VFD load. Now each subsystem $i \in I=\{1,2,3\}$ can be modeled as (7) with states $x_{i}$ and nonlinear system matrix $A_{i}\left(x_{i}\right)$. The input $w_{i}$ and output $v_{i}$ of each subsystem $i \in I$ can be expressed in terms of the node voltage and current states of other subsystems with matrices $B_{i}$ and $C_{i}$.

\section{REFERENCES}

[1] C. Chen, J. Wang, F. Qiu, and D. Zhao, "Resilient Distribution System by Microgrids Formation after Natural Disasters," IEEE Transactions on Smart Grid, vol. 7, no. 2, pp. 958-966, 2016.

[2] Z. Wang and J. Wang, "Self-Healing Resilient Distribution Systems Based on Sectionalization Into Microgrids," IEEE Transactions on Power Systems, vol. 30, no. 6, pp. 3139-3149, nov 2015.

[3] W. Zheng, P. Crossley, B. Xu, and H. Qi, "Transient stability of a distribution subsystem during fault-initiated switching to islanded operation," International Journal of Electrical Power and Energy Systems, vol. 97, no. August 2017, pp. 418-427, 2018.

[4] A. H. Kasem Alaboudy, H. H. Zeineldin, and J. Kirtley, "Microgrid stability characterization subsequent to fault-triggered islanding incidents," IEEE Transactions on Power Delivery, vol. 27, no. 2, pp. 658-669, 2012.

[5] M. H. Roos, P. H. Nguyen, J. Morren, and J. G. Slootweg, "Probabilistic Stability Analysis of Fault-Initiated Islanding Microgrids Without Inertia," Preprints, pp. 1-16, 2020. 
[6] M. Farrokhabadi, C. A. Canizares, J. W. Simpson-Porco, E. Nasr, L. Fan, P. Mendoza-Araya, R. Tonkoski, U. Tamrakar, N. D. Hatziargyriou, D. Lagos, R. W. Wies, M. Paolone, M. Liserre, L. Meegahapola, M. Kabalan, A. H. Hajimiragha, D. Peralta, M. Elizondo, K. P. Schneider, F. Tuffner, and J. T. Reilly, "Microgrid Stability Definitions, Analysis, and Examples," IEEE Transactions on Power Systems, vol. 8950, no. APRIL, pp. 1-1, 2019.

[7] F. Katiraei, M. R. Iravani, and P. W. Lehn, "Micro-grid autonomous operation during and subsequent to islanding process," IEEE Transactions on Power Delivery, vol. 20, no. 1, pp. 248-257, 2005.

[8] IEEE PES Power System Dynamic Performance Committee IEEE and IEEE PES Task Force on Microgrid Stability Analysis and Modeling, "Microgrid Stability Definitions, Analysis and Modeling," Tech. Rep. April, 2018.

[9] D. Marx, P. Magne, B. Nahid-Mobarakeh, S. Pierfederici, and B. Davat, "Large signal stability analysis tools in DC power systems with constant power loads and variable power loads-A review," IEEE Transactions on Power Electronics, vol. 27, no. 4, pp. 1773-1787, 2012.

[10] M. Kabalan, P. Singh, and D. Niebur, "A Design and Optimization Tool for Inverter-Based Microgrids Using Large-Signal Nonlinear Analysis," IEEE Transactions on Smart Grid, vol. 10, no. 4, pp. 4566-4576, jul 2019.

[11] B. Severino and K. Strunz, "Enhancing Transient Stability of DC Microgrid by Enlarging the Region of Attraction Through Nonlinear Polynomial Droop Control," IEEE Transactions on Circuits and Systems I: Regular Papers, vol. 66, no. 11, pp. 4388-4401, 2019.

[12] C. J. Sullivan, S. D. Sudhoff, E. L. Zivi, and H. Ż. Stanislaw, "Methods of Optimal Lyapunov Function Generation with Application to Power Electronic Converters and Systems," pp. 267-274, 2007.

[13] Z. Lendek, T. M. Guerra, R. Babuška, and B. De Schutter, Stability Analysis and Nonlinear Observer Design Using Takagi-Sugeno Fuzzy Models, ser. Studies in Fuzziness and Soft Computing. Berlin, Heidelberg: Springer Berlin Heidelberg, 2011, vol. 262.

[14] M. Kabalan, P. Singh, and D. Niebur, "Large Signal Lyapunov-Based Stability Studies in Microgrids: A Review," IEEE Transactions on Smart Grid, vol. 8, no. 5, pp. 2287-2295, 2017.

[15] J. Schiffer, R. Ortega, A. Astolfi, J. Raisch, and T. Sezi, "Conditions for stability of droop-controlled inverter-based microgrids," Automatica, vol. 50, no. 10, pp. 2457-2469, 2014.

[16] B. Qin, X. Zhang, J. Ma, S. Mei, and D. J. Hill, "Local Input to State Stability Based Stability Criterion with Applications to Isolated Power Systems," IEEE Transactions on Power Systems, vol. 31, no. 6, pp. 5094-5105, 2016.

[17] Y. Xia, W. Wei, T. Long, F. Blaabjerg, and P. Wang, "New Analysis Framework for Transient Stability Evaluation of DC Microgrids," IEEE Transactions on Smart Grid, vol. 11, no. 4, pp. 2794-2804, 2020.

[18] J. C. Willems, "Dissipative Dynamical Systems Part I . General Theory," Archive for Rational Mechanics and Analysis, vol. 45, no. 5, pp. 321351,1972

[19] _ , "Dissipative Dynamical Systems Part II . Linear Systems with Quadratic Supply Rates," Archive for Rational Mechanics and Analysis, vol. 45 , no. 5, pp. 352-393, 1972.

[20] A. Jokic and I. Nakic, "On Additive Lyapunov Functions and Existence of Neutral Supply Rates in Acyclic LTI Dynamical Networks," in 22nd International Symposium on Mathematical Theory of Networks and Systems, Minneapolis, MN, USA, 2016.

[21] _ , "On Structured Lyapunov Functions and Dissipativity in Interconnected LTI Systems," IEEE Transactions on Automatic Control, vol. PP, no. c, pp. 1-1, 2019.

[22] N. Pogaku, M. Prodanović, and T. C. Green, "Modeling, analysis and testing of autonomous operation of an inverter-based microgrid," IEEE Transactions on Power Electronics, vol. 22, no. 2, pp. 613-625, 2007.

[23] H. K. Khalil, Nonlinear Systems, 2001.

[24] L. Herrera, W. Zhang, and J. Wang, "Stability Analysis and Controller Design of DC Microgrids with Constant Power Loads," IEEE Transactions on Smart Grid, vol. 8, no. 2, pp. 881-888, 2017.

[25] M. Arcak, C. Meissen, and A. Packard, Networks of dissipative systems compositional certification of stability, performance, and safety, 2016, no. 9783319299273 .

[26] Z. Liu, M. Su, Y. Sun, W. Yuan, H. Han, and J. Feng, "Existence and Stability of Equilibrium of DC Microgrid With Constant Power Loads," IEEE Transactions on Power Systems, vol. 33, no. 6, pp. 6999-7010, nov 2018.

[27] A. S. Matveev, J. E. Machado, R. Ortega, J. Schiffer, and A. Pyrkin, "A Tool for Analysis of Existence of Equilibria and Voltage Stability in Power Systems With Constant Power Loads," IEEE Transactions on Automatic Control, vol. 65, no. 11, pp. 4726-4740, nov 2020.
[28] J. H. Teng, "A direct approach for distribution system load flow solutions," IEEE Transactions on Power Delivery, vol. 18, no. 3, pp. 882-887, 2003.

[29] D. Dong, B. Wen, D. Boroyevich, P. Mattavelli, and Y. Xue, "Analysis of Phase-Locked Loop Low-Frequency Stability in Three-Phase Grid-Connected Power Converters Considering Impedance Interactions," IEEE Transactions on Industrial Electronics, vol. 62, no. 1, pp. 310-321, jan 2015.

[30] M. H. Roos, P. H. Nguyen, J. Morren, and J. G. Slootweg, "Aggregation of Component-Based Grid-Feeding DER and Load Models for Simulation of Microgrid Islanding Transients," in Proceedings of the 21 st Power Systems Computation Conference, Porto, Portugal, 2020.

[31] — , "Modeling and Experimental Validation of Power Electronic Loads and DERs For Microgrid Islanding Simulations," IEEE Transactions on Power Systems, vol. 35, no. 3, pp. 2279-2288, may 2020.

[32] A. K. Ghosh, D. L. Lubkeman, M. J. Downey, and R. H. Jones, "Distribution circuit state estimation using a probabilistic approach," IEEE Power Engineering Review, vol. 17, no. 2, pp. 46-47, 1997.

[33] M. Amin and Q.-C. Zhong, "Resynchronization of Distributed Generation Based on the Universal Droop Controller for Seamless Transfer Between Operation Modes," IEEE Transactions on Industrial Electronics, vol. 67, no. 9, pp. 7574-7582, 2019.

[34] Z. Shuai, C. Shen, X. Yin, X. Liu, and Z. J. Shen, "Fault Analysis of Inverter-Interfaced Distributed Generators With Different Control Schemes," IEEE Transactions on Power Delivery, vol. 33, no. 3, pp. 1223-1235, jun 2018

[35] Z. Shuai, Y. Peng, J. M. Guerrero, Y. Li, and Z. J. Shen, "Transient Response Analysis of Inverter-Based Microgrids Under Unbalanced Conditions Using a Dynamic Phasor Model," IEEE Transactions on Industrial Electronics, vol. 66, no. 4, pp. 2868-2879, apr 2019.

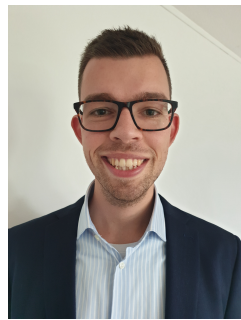

Martijn Roos (S'17) received the B.Eng. degree in electrical engineering from HAN University of Applied Sciences, Arnhem, the Netherlands in 2014 and the M.Sc. degree in electrical engineering from Eindhoven University of Technology, Eindhoven, the Netherlands in 2017, where he is currently working towards the Ph.D. degree on the dynamics and control of microgrids during islanding transitions at the Electrical Energy Systems group. His main research interests include: modeling and dynamics of microgrids, real-time control of distributed energy resources, power system simulation and stability analysis, and adaptive protection systems.

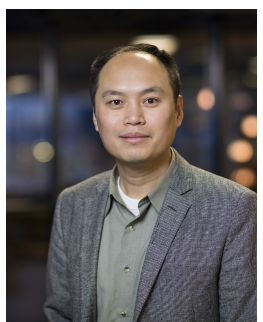

Phuong Nguyen (M'06) received the Ph.D. degree from the Eindhoven University of Technology, the Netherlands in 2010. Before joining the Environmental Research and Innovation (ERIN) department of the Luxembourg Institute of Science and Technology (LIST) in 2019 as the group leader of the Sustainable Energy Systems (SES) group, Dr. Nguyen has been holding an associate professor position with tenure at the Electrical Energy System (EES) group, Eindhoven University of Technology (TU/e). He was a visiting researcher with the Real-Time Power and Intelligent Systems (RTPIS) Laboratory, Clemson University, USA, in 2012 and 2013. Dr. Nguyen has committed his research effort to realize synergies of advanced monitoring and control functions for the distribution networks along with emerging digital technologies. This distinctive combination of competences allows him to develop a research pathway crossing over various domains of mathematical programming, stochastics, data mining, and communication networks. His research of interests includes data analytics with deep learning, real-time system awareness using (IoT) data integrity, as well as predictive and corrective grid control functions. 


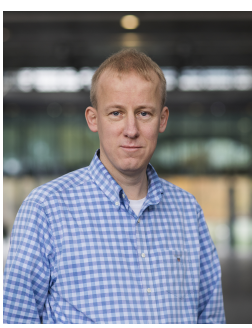

Johan Morren (M'03) received the M.Sc. and Ph.D degree in Electrical Power Engineering both from the Delft University of Technology, The Netherlands in 2000 and 2006 respectively. Since 2006 Johan is network strategist with the Asset Management department of Enexis B.V., one of the largest Distribution Grid Operators of the Netherlands. His main areas of interest are protection and automation, network planning, and distributed generation. He is also associate professor (part-time) at the Electrical Energy Systems group at the Eindhoven University of Technology, the Netherlands.

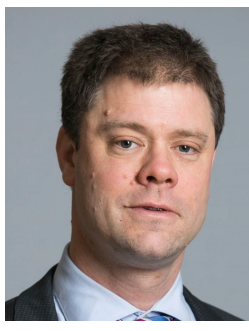

J.G. (Han) Slootweg (M'00; SM'19) received the M.Sc. degree in electrical power engineering in 1998 (cum laude) and the Ph.D. degree in 2003, both from Delft University of Technology, Delft, The Netherlands. He also received the M.Sc. degree in business administration. He is currently Director of the Asset Management Department of Enexis Netbeheer B.V., Hertogenbosch, The Netherlands, one of the largest Distribution Network Operators of the Netherlands. Its spearheads are the strategic goals of Enexis: accelerating the transition towards a more sustainable energy supply and excellent, state of the art network operation. Han also holds a professorship in Smart Grids at the Electrical Energy Systems group at the Eindhoven University of Technology. He has (co-)authored more than 200 papers, covering a broad range of various aspects of electrical power systems. 